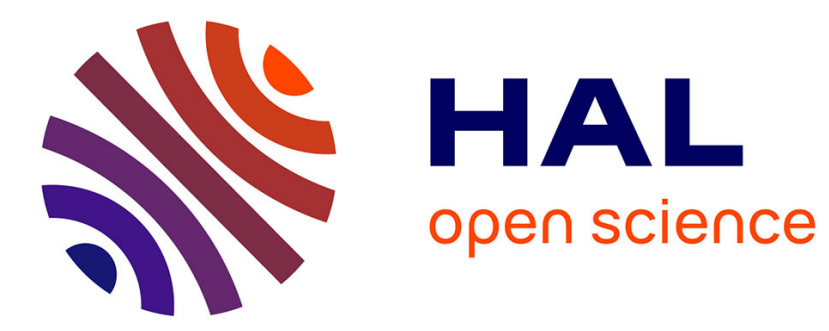

\title{
Photoluminescence of Si nanocrystals under selective excitation
}

\author{
D. Dobrovolskas, J. Mickevičius, G. Tamulaitis, V. Reipa
}

\section{To cite this version:}

D. Dobrovolskas, J. Mickevičius, G. Tamulaitis, V. Reipa. Photoluminescence of Si nanocrystals under selective excitation. Journal of Physics and Chemistry of Solids, 2009, 70 (2), pp.439. 10.1016/j.jpcs.2008.11.013 . hal-00508473

\section{HAL Id: hal-00508473 \\ https://hal.science/hal-00508473}

Submitted on 4 Aug 2010

HAL is a multi-disciplinary open access archive for the deposit and dissemination of scientific research documents, whether they are published or not. The documents may come from teaching and research institutions in France or abroad, or from public or private research centers.
L'archive ouverte pluridisciplinaire $\mathbf{H A L}$, est destinée au dépôt et à la diffusion de documents scientifiques de niveau recherche, publiés ou non, émanant des établissements d'enseignement et de recherche français ou étrangers, des laboratoires publics ou privés. 


\section{Author's Accepted Manuscript}

Photoluminescence of Si nanocrystals under selective excitation

D. Dobrovolskas, J. Mickevičius, G. Tamulaitis, V. Reipa

PII: S0022-3697(08)00540-4

DOI:

Reference: doi:10.1016/j.jpcs.2008.11.013 PCS 5755

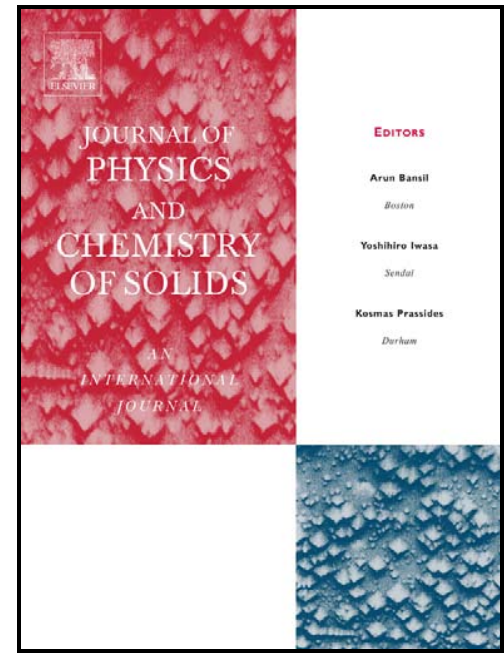

www.elsevier.com/locate/jpcs

To appear in: $\quad$ Journal of Physics and Chemistry of Solids

Received date: $\quad 25$ August 2008

Revised date: $\quad 16$ October 2008

Accepted date: 19 November 2008

Cite this article as: D. Dobrovolskas, J. Mickevičius, G. Tamulaitis and V. Reipa, Photoluminescence of Si nanocrystals under selective excitation, Journal of Physics and Chemistry of Solids (2008), doi:10.1016/j.jpcs.2008.11.013

This is a PDF file of an unedited manuscript that has been accepted for publication. As a service to our customers we are providing this early version of the manuscript. The manuscript will undergo copyediting, typesetting, and review of the resulting galley proof before it is published in its final citable form. Please note that during the production process errors may be discovered which could affect the content, and all legal disclaimers that apply to the journal pertain. 


\title{
Photoluminescence of Si nanocrystals under selective
}

\section{excitation}

D. Dobrovolskas ${ }^{\mathrm{a},{ }^{,}}$, J. Mickevičius $^{\mathrm{a}},{ }^{\text {G. Tamulaitis }}{ }^{\mathrm{a}}$ and V. Reipa ${ }^{\mathrm{b}}$

${ }^{a}$ Institute of Materials Science and Applied Research and Semiconductor Physics

Department, Vilnius University, LT-10222 Vilnius, Lithuania

${ }^{\mathrm{b}}$ Biotechnology Division, National Institute of Standards and Technology, Gaithersburg, Maryland 20899-8312, USA

\begin{abstract}
Photoluminescence of Si nanocrystals passivated by different alkanes (hexane, octane, and tridecane) was studied at room temperature. It is shown that the emission band shape is not affected by the length of the carbon chain in the alkanes used for passivation. A pronounced fine structure of the photoluminescence band consisting of peaks separated by $150-160 \mathrm{meV}$ was observed under resonant excitation. The structure is interpreted by predominant contribution from Si nanocrystal groups with particular stable size/shape existing in addition to the previously reported nanocrystals with "magic" numbers of Si atoms. The contribution of these stable nanocrystals is revealed using selective resonant photoexcitation to the higher energy states in the discrete energy spectrum of such nanocrystals.

${ }^{*}$ Corresponding author. Tel.: +370 52366070 ; fax: +370 52366059 .

E-mail address: darius.dobrovolskas@ff.vu.lt (D. Dobrovolskas)
\end{abstract}


PACS: 78.67.Hc; 81.07.Ta; 78.55.Ap

Keywords: A. Nanostructures; A. Semiconductors; D. Luminescence;

\section{Introduction}

Semiconductor nanocrystals (quantum dots) have size-dependent physical properties due to quantum confinement of charge carriers. Their light emission with wavelengths tunable by changing the nanocrystal size is attractive for various applications including fluorescent markers. Currently, biological and medical fluorescent imaging technologies predominantly use organic dye based fluorescent markers. However, the organic dyes suffer from inherent emission instability under extended excitations. Moreover, their emission bands are broad and simultaneous detection of signals from several markers (multiplexing) is complicated due to spectral overlap [1]. Next generation markers, which are based on II-VI or III-V semiconductor nanocrystals (NCs), demonstrates significant advantages over the organic dyes [1,2]. Their broad excitation spectrum and narrow emission bands are favorable for the development of biological assays with multiplex detection. Furthermore, high quantum yield, resistance to photo-bleaching, and long-term stability are also significant advantages of the semiconductor NCs as markers. However, the bio-applications of binary semiconductor quantum dots are hampered by their inherent chemical toxicity $[3,4,5]$.

Silicon nanocrystals, along with $\mathrm{C}$ - and $\mathrm{SiC}$-based nanoparticles, are considered to be bio-compatible and are being actively tested for biological labeling $[6,7,8]$. Soon after the first report on the light emission in visible from porous Si [9], free Si NCs were prepared [10]. Considerable research efforts have been devoted to the fundamental and applied aspects of their strong photoluminescence, which was rather unanticipated given the indirect band gap of bulk silicon [11]. Recently, the quantum yield of a single 
hydrogen-capped Si NC as high as $88 \%$ was achieved [12] and rivals the efficiency of the familiar dye fluorescein [13], though the yield of an ensemble of Si NCs is still considerably lower $(\sim 10 \%)[14,15]$. Thus, influence of $\mathrm{NC}$ environment, control of the particle size distribution and related issues require further studies to improve the emission efficiency of the Si NC systems and to ensure tunable emission in a wide spectral range.

Passivation of nanocrystal surface is of primary importance for effective light emission. It is known that hydrogen-passivated Si NCs can be obtained after etching Si in HF based solutions, but are stable only for a short period of time [11]. In order to ensure the long-term PL stability, hydrogen has to be substituted with a more robust capping material $[16,17]$. It is also known that surface passivation might significantly affect the electronic structure and, consequently, the effective band gap of Si NC. For example, small hydrogen-terminated Si NCs emit in the blue, while the PL of the oxygenterminated NCs of the same size peaks in the green-yellow spectral range [18].

This work was aimed at a detailed study of Si NC emission using selective (resonant) photoexcitation in the spectral range overlapping with the emission band. Si NCs passivated by alkanes with different carbon chain length (hexane, octane or tridecane) were prepared for the study. We focused on investigating the emission spectrum dynamics while selecting the excitation photon energies in the close vicinity to the lowest optical transitions of Si NCs. This approach enabled us to reveal new subbands in the photoluminescence (PL) spectra caused by groups of particular stable nanocrystal size/shape that exists in addition to previously reported nanocrystal fractions with the "magic" Si atoms numbers [19].

\section{Experimental}


Samples were prepared by lateral electrochemical etching of a $p$-type (B-doped) silicon wafer $(\mathrm{R}=3-5 \mathrm{ohm} / \mathrm{cm})$. The anodic etch was carried out in the peroxide-HFethanol $\left(\mathrm{H}_{2} \mathrm{O}_{2}: \mathrm{HF}: \mathrm{C}_{2} \mathrm{H}_{5} \mathrm{OH}\right)$ solution at a constant current, which resulted in a nanoporous Si layer. After etching, the wafer was rinsed with deionized water and methanol and blow-dried in a stream of nitrogen to prevent oxidation. The etched wafer was immediately transferred into pure deaerated hexene, octene or tridecene (Sigma-Aldrich, St. Louis, MO) under nitrogen blanket, and sonicated in an ultrasound bath for $1 \mathrm{~h}$. Following filtering through $0.2 \mu \mathrm{m}$ syringe filters, the colloidal suspensions of Si NCs were placed in a quartz container that was mounted inside a UV reactor (model RMR600, Rayonet, Branford, CT) and exposed to $254 \mathrm{~nm}$ light for $30 \mathrm{~min}$. Solvents were subsequently evaporated in a stream of nitrogen and dry nanocrystals were resuspended in carbon tetrachloride.

The PL was excited by the frequency-tripled radiation of a Q-switched YAG:Nd laser $(354 \mathrm{~nm}, 3.494 \mathrm{eV})$ or tunable-wavelength radiation of a dye laser pumped by the YAG:Nd laser. A He-Cd laser $(325 \mathrm{~nm}, 3.81 \mathrm{eV})$ was used for excitation in CW mode. The PL emission was analyzed by a double grating monochromator (Jobin Yvon, HRD1) and detected by a photomultiplier (Hamamatsu, R1463P) in combination with boxcar integrator (when PL was excited with a pulsed laser) or photon counter (when PL was excited with a CW laser). Application of double monochromator with high signal to background contrast enabled us to measure PL spectra in close vicinity to laser line and check for a possible influence of Raman scattering. The PL spectra were digitally processed to account for the spectral response of the detection system. Raman scattering measured in pure solvent had intensity considerably lower than the PL intensity under study, so that the Raman scattering in solvent could not significantly affect the PL spectra. 


\section{Results and discussion}

Figure 1 shows PL spectra of three Si NC colloidal samples containing Si NCs of similar size, but surface-alkylated with hexane (C6), octane (C8), and tridecane (C13). All three samples were dissolved in carbon tetrachloride. The spectra were recorded using excitation by a CW He-Cd laser (excitation photon energy $h v_{\mathrm{exc}}=3.81 \mathrm{eV}$ ). PL spectra of all three samples consist of one broad band peaked at $3.50 \mathrm{eV}$. The similarity of the PL spectra indicates that the emission band shape is not affected by the alkyl chain length used for surface passivation. This experimental observation is in consistence with the calculations indicating a weak dependence of the Si nanocrystal optical bandgap on the length of the passivating alkyl chain [20].

PL spectra of Si nanocrystals passivated by octane (C8) and tridecane (C13) under excitation by photons with energy $\left(h v_{\mathrm{exc}}=3.49 \mathrm{eV}\right)$ close to the emission band are presented in Fig. 2. The major feature of these spectra is the appearance of a wellresolved sub-band structure that was not observed under nonresonant excitation (see Fig. 1). Note that the positions of the sub-bands are not affected by the chain length of the alkyl used for passivation. Nanocrystal surface passivation by different alkyls affects only the relative intensities of the photoluminescence sub-bands. Measurements in the antiStokes range showed no signal detectable above the noise level.

A multipeak PL spectrum with peak separation similar to that observed in our study has been recently reported in single silicon naocrystals grown by laser pyrolysis [21]. However, those satellite bands were shown to be phonon replicas due to phonon modes in $\mathrm{SiO}_{2}$, which typically occurs around the core of $\mathrm{Si}$ nanocrystals fabricated by pyrolysis, but is hardly expected in our samples prepared by electrochemical etching and capped with alkanes. Phonon replicas due to emission of phonons in silicon have been 
observed in PL spectra, however, only at low temperatures [22-24]. Replicas due to emission of the bulk-like transverse optical (TO, $57.3 \mathrm{meV}$ ), longitudinal optical (LO, $55.3 \mathrm{meV}$ ), and transverse acoustic (TA, $18.2 \mathrm{meV}$ ) phonons have been identified. However, none of the phonon energies either their combination match the separation between the peaks of the PL features observed in our experiments. Moreover, the reported phonon replicas were observed only at low temperatures $(<70 \mathrm{~K})$, while our measurements were performed at room temperature. Consequently, the PL spectral structure observed in the present work cannot be interpreted by phonon replicas.

To observe dynamics of the sub-band structure, PL was studied in detail under excitation with tunable-wavelength emission of a dye laser. Dynamics of PL spectrum at variable excitation wavelength is illustrated for sample C8 in Fig. 3. Nanocrystals passivated with other alkyls showed a similar dynamics. Several distinct sub-bands are clearly resolved in each of the spectra shown in Fig. 3. Fitting of the spectrum with superposition of Gaussian-shape sub-bands shows that the positions of the sub-bands do not depend on the excitation wavelength. As demonstrated in Fig. 3 for the spectra measured at different excitation photon energies, the experimental spectra can be fitted using a superposition of Gaussian sub-bands with fixed positions. Only the sub-band intensities were allowed to vary in the fitting. The sub-band peak positions and their separations are listed in Table 1. To the best of our knowledge, these features were not observed in $\mathrm{Si}$ nanocrystals before. As discussed in more detail below, resonant excitation was crucial for detection of these features.

The sub-band structure of the PL spectrum under resonant excitation might be related to prevailing contribution by Si NCs of certain sizes. Energy of the lowest optical transition $\Delta E$ (effective band gap) in nanocrystals with pronounced quantum confinement strongly depends on the nanocrystal diameter $d$. This dependence in Si NCs 
is somewhat weaker than that for an ideal electron-hole pair in a spherical nanocrystal $\left(\Delta E \propto d^{-2}\right)$ and is described by a function $\Delta E \propto d^{-\alpha}$ with $\alpha$ values of 0.5 [19], 1.34 [25], 1.39 [26], or 1.39 with an additional $1 / d$ term [26]. A varying degree of size dispersity is typical for Si NC preparation. Consequently, the optical transitions have considerable inhomogeneous broadening. In addition, predominant occurrence of nanocrystals with certain diameters has been reported. Patrone et al. [25] observed a structure in the PL band of silicon nanoclusters at room temperature and proposed a discrete size model interpreting the structure by predominant contribution of nanocrystals with a complete outer atomic layer. The incomplete outer layers cause formation of nonradiative recombination centers that quench PL of the Si NCs. According to this model, the adjacent radii of nanocrystals, which predominantly contribute to the PL of the entire ensemble, should differ by the thickness of one Si monolayer $(0.314 \mathrm{~nm})$. The corresponding $\mathrm{NC}$ diameters generate the following series: 1.300, 1.928, 2.556, 3.184, and $3.812 \mathrm{~nm}$ [25]. On the other hand, calculations show that clusters of $\mathrm{Si}$ atoms containing complete atomic shells correspond to stabilized nanostructures $[27,28]$. Based on high-resolution TEM measurements, hydrogen terminated Si NCs were classified into several discrete size groups containing NCs with diameters of 1.0, 1.67, 2.15, 2.9 and $3.7 \mathrm{~nm}$. These Si nanoparticles containing so-called "magic" numbers of atoms and emit at $3.44,2.64,2.39$, and $2.11 \mathrm{eV}$, respectively [19].

We observe new discrete spectral features at photon energies in the range flanked by the effective band gaps of the two smallest nanocrystals with the "magic" numbers of Si atoms (diameters 1.0 and $1.67 \mathrm{~nm})$. The spectral separation of these features $(\sim 150$ $160 \mathrm{meV}$, see Table 1) is considerably smaller than that between the structures observed due to existence of the "magic" NCs before. The separation is similar to the degeneracy of the order of 0.1-0.2 eV, which has been calculated for Si NC isomers containing 30-40 
atoms [27,29]. Thus, we believe that the new fine structure observed in our PL emission spectra originates from the predominant contribution of certain nanocrystals with increased stability due to their specific surface configuration (shape), since various stable shapes and surface atom configurations are reported for Si nanocrystals [19,30,31]. Since our nanocrystals were produced by splitting bulk silicon crystal, it is reasonable to expect that the nanocrystal internal structure retains that of the bulk material. However, given significant differences in the isomer chemical reactivity [29], the Si nanocrystal surface might be further altered during the photo-assisted hydrosilylation reaction. Consequently, nanocrystals with particular shapes and surface configurations can dominate in a nanocrystal ensemble with fixed Si atom number.

Due to the high density of the higher excited energy states, photons with energies considerably exceeding the effective band gap of the nanocrystals can be quite effectively absorbed by nanocrystals of any size/configuration. Meanwhile, excitation by photons with energies in the vicinity of the emission band results in selective excitation of certain nanocrystals having energy levels in resonance with the excitation photon energy. Tuning the excitation wavelength allows one to selectively enhance the contribution of a certain group of stable nanocrystals to the total emission spectrum, as it has previously been demonstrated for CdSe nanocrystals [32]. This situation is schematically explained in Fig. 4, where Si NC emission spectra measured at different excitation wavelengths (indicated by horizontal arrows) are plotted. In Fig. 4(b), the calculated energies of the lowest optical transitions are plotted versus nanocrystal radius by a bold solid line using the expression presented in [19]. The effective band gaps of two full-atom-shell nanocrystals containing subsequent "magic numbers" [19] are indicated as open circles on the bold line. The upper curves represent the NC radius dependences for the energies of the higher optical transitions, which are plotted using the approach of Allan et al. [33]. 
Although the accuracy of the higher optical transition energy calculations is considerably lower than that for the lowest transition, the plot in Fig. 4(b) demonstrates how excitation via these higher energy states facilitates predominant selective excitation of nanocrystals having certain energy spectrum. Photons with energy $h v_{\text {exc }}$ preferably excite those nanocrystals, which have upper energy levels in resonance with $h v_{\text {exc }}$. The photoexcited carriers relax to the lowest energy state and their radiative recombination results in emission of photons with energies corresponding to the effective band gap in the nanocrystal. Consequently, the resonantly excited nanocrystals will have an enhanced contribution to the total emission spectrum of the $\mathrm{NC}$ ensemble. In a nanocrystal ensemble with continuous size distribution, shifting the excitation photon energy would continuously shift the PL band peak position through the homogeneously broadened total emission spectrum. In case of predominant existence of NCs with specific energy spectrum (due to specific size or/and shape configuration), as in the ensemble of Si NCs under study, the selective excitation enhances contribution of a certain NC group. Thus, the resonant excitation reveals the structured shape of the total emission spectrum, which gets leveled when excited by higher energy photons. Note that tuning the excitation photon energy does not affect the spectral positions of the sub-bands in the total emission spectrum, since the positions depend on the predominant effective bandgaps of the corresponding nanocrystal groups. Meanwhile, intensities of the sub-bands depend on excitation wavelength due to the resonant excitation.

\section{Conclusions}

In conclusion, passivation of $\mathrm{Si}$ nanocrystal surface by alkanes with different carbon chain length does not significantly affect the emission spectrum of the 
nanocrystals. Application of the resonant excitation enabled us to reveal new distinct subbands in the emission spectrum of the alkylated Si nanocrystals. The peaks of these subbands are separated by $150-160 \mathrm{meV}$ and are not affected either by the alkyl carbon chain length, or by variation in the excitation wavelength. The fine structure of the emission spectra observed in our resonant PL experiments is caused by predominant contribution of nanocrystals with specific number of atoms and surface configuration (shape). Optimization of conditions for formation of such stable and emission-effective $\mathrm{Si}$ nanocrystals might increase the quantum yield of the Si NC markers.

\section{Acknowledgement}

We gratefully acknowledge support from the NATO Public Diplomacy Division. 


\section{References}

[1] I.L. Medintz, H.T. Uuyeda, E.R. Goldman, H. Mattoussi, Nature Materials 4 (2005) $435-446$.

[2] C.J. Murphy, Anal. Chem. 74 (2002) 521-526.

[3] C. Kirchner, T. Liedl, S. Kudera, T. Pellegrino, A.M. Javier, H.E. Gaub, S. Stolzle, N. Fertig, W.J. Parak, Nano Lett. 5 (2005) 331-338.

[4] A.M. Derfus, W.C. W. Chan, S.N. Bhatia, Nano Lett. 4 (2004) 11-18.

[5] R. Hardman, Environ. Health Perspect. 114 (2006) 165-172.

[6] L. Wang, V. Reipa, J. Blasic, Bioconjugate Chem. 15 (2004) 409-412.

[7] J. Choi, N.S. Wang, V. Reipa, Bioconjugate Chem. 19 (2008) 680-685.

[8] N. O' Farrell, A. Houlton, B.R. Horrocks, Intern. J. Nanomed. 1 (2006) 451-459.

[9] L.T. Canham, Appl. Phys. Lett. 57 (1990) 1046-1048.

[10] J.L. Heinrich, C.L. Curtis, G.M. Credo, K.L. Kavanagh, M.J. Sailor, Science 255 (1992) 66-68.

[11] A.G. Cullis, L.T. Canham, P.D.J. Calcott, J. Appl. Phys. 82 (1997) 909-965.

[12] M. Credo, M.D. Mason, S.K. Buratto, Appl. Phys. Lett. 74 (1999) 1978-1982.

[13] O. Akcakir, J. Therrien, G. Belomoin, N. Barry, J.D. Muller, E. Gratton, M. Nayfeh, Appl. Phys. Lett. 16 (2000) 1897-1859.

[14] A. Fojtik, A. Henglein, J. Phys. Chem. B, 110 (2006) 1994-1998.

[15] D.S. English, L.E. Pell, Z. Yu, P.F. Barbara, B.A. Korgel, Nano Lett. 2 (2002) 681685.

[16] J.M. Buriak, Chem. Commun. 12 (1999) 1051-1060.

[17] R. Boukherroub, S. Morin, D.M. Wayner, F. Bensebaa, G.I. Sproule, J.M. Baribeau, D.J. Lockwood, Chem. Mater. 13 (2001) 2002-2011. 
[18] Z. Zhou. L. Brus, R. Friesner, Nano Lett. 3 (2003) 163-167.

[19] G. Belomoin, J. Therrien, A. Smith, S. Rao, R. Twestwn, S. Chaieb, M. Nayfeh,

L. Wagner, L. Mitas, Appl. Phys. Lett. 80 (2002) 841-843.

[20] F.A. Roboredo, G. Galli, J. Phys. Chem. B 109 (2005) 1072-1078.

[21] J. Martin, F. Cichos, F. Huisken, C. Borczyskowski, Nano Lett. 8 (2008) 656-660.

[22] T. Seumoto, K. Tanaka, A. Nakajima, T. Itakura, Phys. Rev. Lett. 70 (1993) 36593662.

[23] M. Rosenbauer, S. Finkbeiner, B. Bustarret, J. Weber, Phys. Rev B 51 (1995) $10539-10545$.

[24] Y. Kanemitsu, S. Okamoto, Phys. Rev. B. 58 (1998) 9652-9655.

[25] L. Petrone, D. Nelson, V.I. Safarov, M. Sentis, W. Marine, J. Appl. Phys. 87 (2000) $3829-3837$.

[26] G. Ledoux, O. Guillois, D. Porterat, C. Reynaud, F. Huisken, B. Kohn, V. Paillard, Phys. Rev. B 63 (2000) 942-950 .

[27] U. Rothlisberger, W. Andreoni, M. Parinello, Phys. Rev. Lett. 72 (1994) 665-668.

[28] L.A. Bloomfield, R.R. Freeman, W.L. Brown, Phys. Rev. Lett. 54 (1985) 22462249.

[29] M.F. Jarrold, E.C. Honea, J. Am. Chem. Soc. 114 (1992) 459-464.

[30] E.W. Draeger, J.C. Grossman, A.J. Williamson, G. Galli, Phys. Rev. Lett 90 (2003) $167402-167406$.

[31] Y. Zhao, Yong-Hyun Kim, Mao-Hua Du, S.B. Zhang, Phys. Rev. Lett 93 (2004) 015502-015505.

[32] P.A.M. Rodrigues, G. Tamulaitis, P.Y. Yu, S.H. Risbud, Solid State Commun. 94 (1995) 583-587.

[33] G. Allan, C. Delerue, Phys. Rev. B. 66 (2002) 233303-233306. 


\section{Figure captions}

Fig. 1. PL spectra of $\mathrm{Si}$ nanocrystal suspensions in $\mathrm{CCl}_{4}$ passivated with hexane (C6), octane (C8), and tridecane (C13). Spectra are normalized and offset on the vertical axis for clarity. Excitation photon energy $h v_{\mathrm{exc}}=3.81 \mathrm{eV}$.

Fig. 2. Resonant PL spectra of Si nanocrystals passivated by octane (C8) and tridecane (C13). Excitation photon energy $h v_{\mathrm{exc}}=3.49 \mathrm{eV}$.

Fig. 3. PL spectra of octane-passivated Si nanocrystals at several excitation photon energies (dots). Solid lines represent fits by a set of Gaussian bands shown at the bottom. Spectra are vertically offset for clarity.

Fig. 4. Resonant PL spectra of the octane-passivated Si NCs (a) and nanocrystal diameter dependence of energy levels in Si NCs (b): for effective band gap (solid bold line, calculated as in [32]) and for two higher excited states (narrow lines, calculated as in [19]). The horizontal dashed lines are eye-guides. Excitation photon energies used in the experiment are indicated as horizontal arrows. 
Table 1. PL peak positions and their separations in a multi-Gaussian fit for PL spectra shown in Fig. 3.

\begin{tabular}{ll}
\hline Peak position $(\mathrm{eV})$ & Difference $(\mathrm{meV})$ \\
\hline 2.596 & 155 \\
2.751 & 152 \\
2.903 & 164 \\
3.067 & 155 \\
3.221 & 159 \\
3.381 & 167 \\
3.547 & \\
\hline
\end{tabular}


Fig. 1

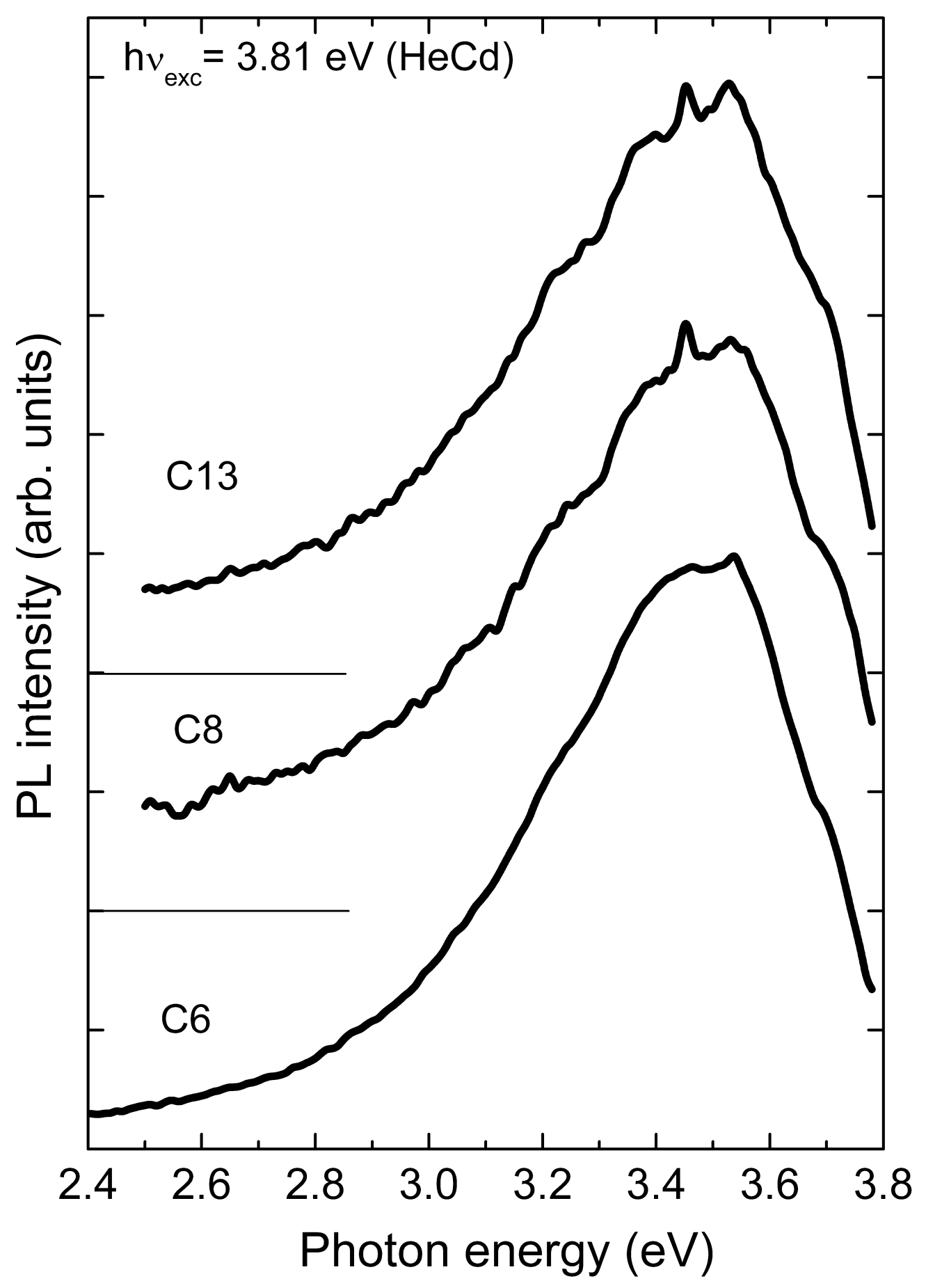


Fig. 2

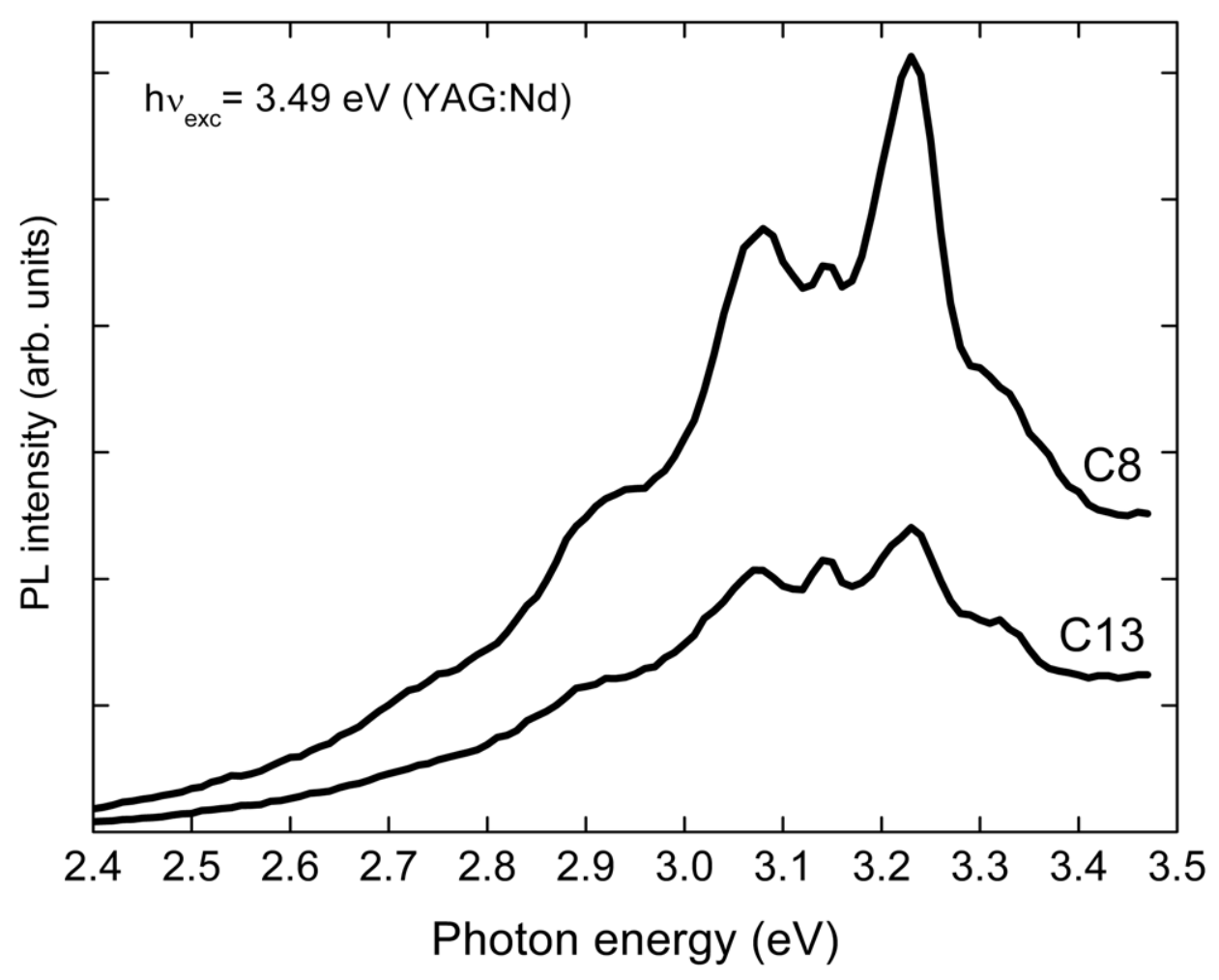


Fig. 3

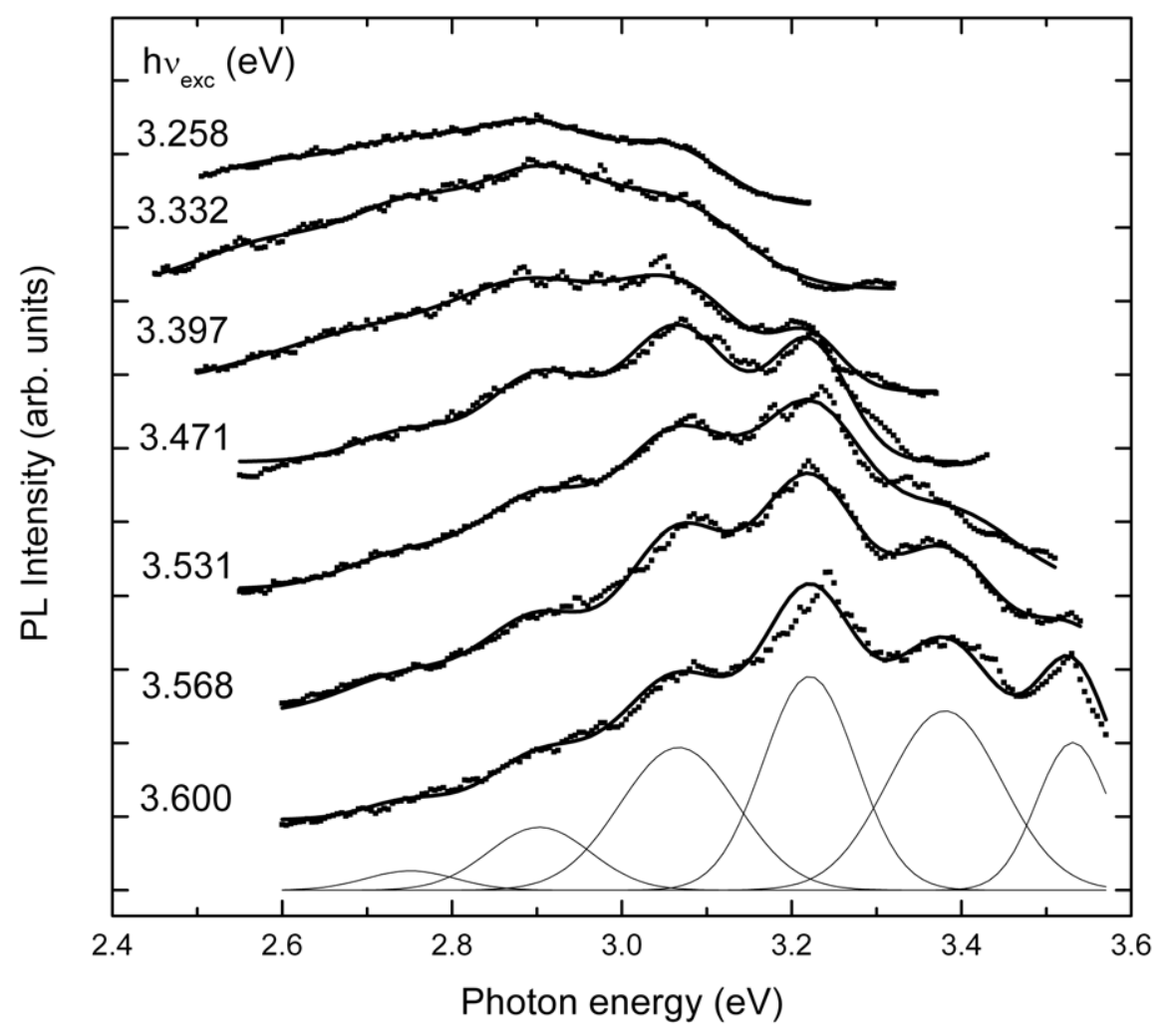


Fig. 4

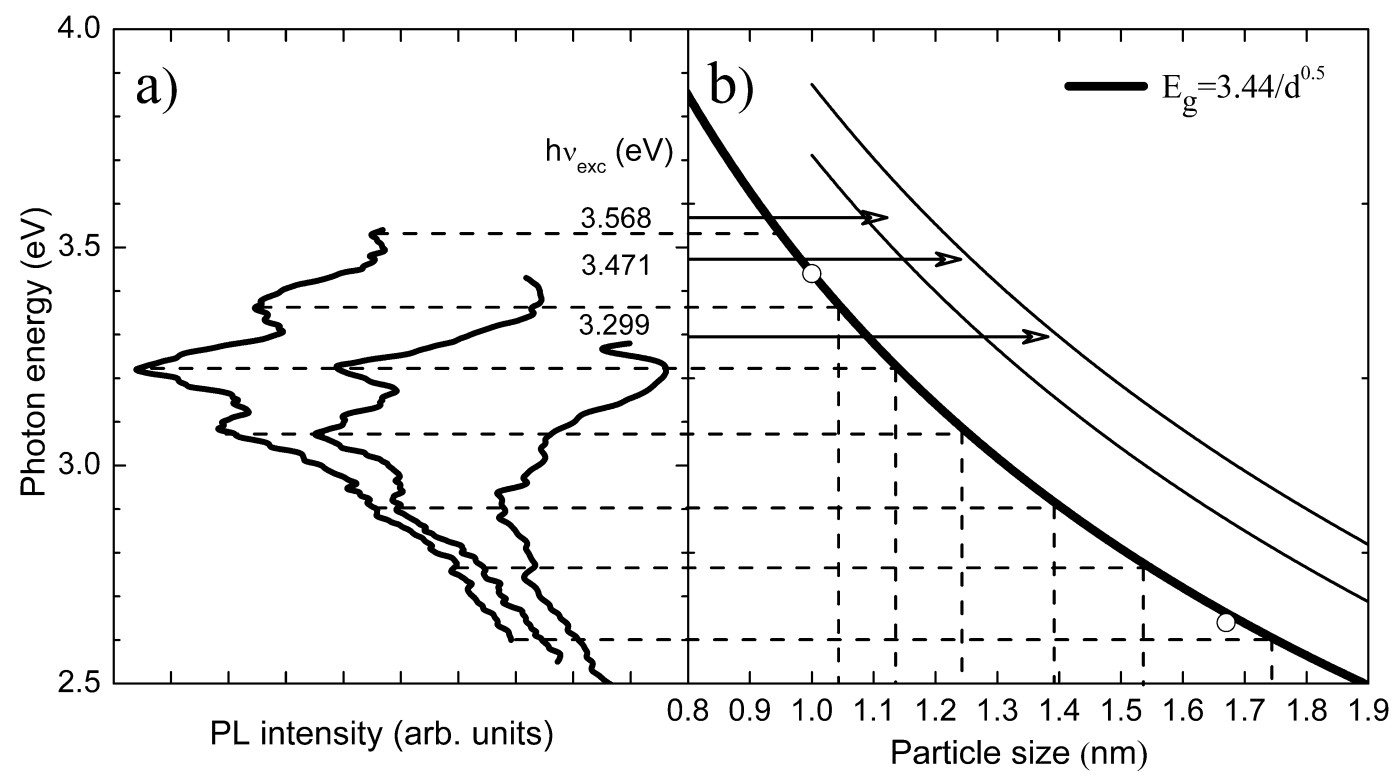

